\title{
On the One Extremal Problem with the Free Poles on the Unit circle
}

\author{
Andrey L. Targonskii \\ Zhytomyr Ivan Franko State University, Ukraine \\ targonsk@mail.ru, targonsk@zu.edu.ua
}

Keywords: inner radius of domain, quadratic differential, piecewise-separating transformation, Green function, radial systems of points, logarithmic capacity, variational formula.

Abstract. The sharp estimates of the product of the inner radius for pairwise disjoint domains are obtained. In particular, we solve an extremal problem in the case of an arbitrary finite number of the free poles on the unit circle for the following functional

$$
\prod_{k=1}^{n}\left(\left|a_{k+1}-a_{k}\right|^{\alpha} \cdot r\left(B_{k}, a_{k}\right)\right) \text {. }
$$

\section{Introduction}

This paper is belong to the theory of extremal problems on the classes of non-overlapping domain, which is a separate direction in geometric theory of functions of a complex variable. The begin of these investigations is associated with the paper of M. A. Lavrent'ev [1] in 1934. He found the maximum of some functional with respect to two simply connected domains with two fixed points. We note that this result was needed him for applying to some aerodynamics problems. In 1947, G. M. Goluzin solved a similar problem for three fixed points on the complex plane [2]. Then the topic began to evolve rapidly. In this connection we may recall the papers of many authors, including Y. E. Alenitsina, M. A. Lebedev, J. Jenkins, P. M. Tamrazov, P. P. Kufareva and others. Using the idea of P. M. Tamrazov, in 1975 G. P. Bakhtin solved first the problem with so-called "free poles" on the unit circle, see, e.g., [3].

An important step for the development of this topic was the papers of V. N. Dubinin. He developed a new method of research that is the method of piecewise-separating transformation. He also first solved numerous of extremal problems for the arbitrary but fixed multi connected non-overlapping domains (see, e.g., [4]- [6]). Now this type of extremal problems is used for the investigations in the holomorphic dynamics.

In the last decade actively used Bakhtin's method of "managing functional". He managed to solve a series of extremal problems for so-called "radial systems of points" (see, e.g., [4], [7]-[12]). In the present paper we use the mentioned above Bakhtin's method.

The subject of studying of our work is the following problem.

Let $n \in \mathbb{N}, n \geq 2, \alpha \geq 0$. The maximum of the functional be found

$$
\prod_{k=1}^{n}\left(\left|a_{k+1}-a_{k}\right|^{\alpha} \cdot r\left(B_{k}, a_{k}\right)\right)
$$

where $A_{n}=\left\{a_{k}\right\}_{k=1}^{n}$ - an arbitrary system of the points on the unit circle, $\left\{B_{k}\right\}_{k=1}^{n}$ - an arbitrary set of the non-overlapping domains, $a_{k} \in B_{k} \subset \overline{\mathbb{C}}(k=\overline{1, n})$.

\section{Theory}

Let $\mathbb{N}, \mathbb{R}$ are the sets of the natural and real numbers of conformity, $\mathbb{C}$ - the plain of the complex numbers, $\overline{\mathbb{C}}=\mathbb{C} \bigcup\{\infty\}$ - the Riemannian sphere. 
For the fix number $n \in \mathbb{N}$ and the system of the points

$$
A_{n}=\left\{a_{k}\right\}_{k=1}^{n}
$$

the relations are executed:

$$
\begin{gathered}
0=\arg a_{1}<\arg a_{2}<\ldots<\arg a_{n}<2 \pi, \\
a_{k} \in \mathbb{C}, \quad\left|a_{k}\right|=1, k=1,2, \ldots, n .
\end{gathered}
$$

For such systems of points we will consider the following sizes:

$$
\sigma_{k}=\frac{1}{\pi}\left(\arg a_{k+1}-\arg a_{k}\right), k=1,2, \ldots, n, \quad a_{n+1}:=a_{1} .
$$

Let's consider the system of the angular domains:

$$
M_{k}:=\left\{w: \arg a_{k}<\arg w<\arg a_{k+1}\right\}, \quad k=\overline{1, n}, \quad a_{n+1}:=a_{1}
$$

Let $\left\{B_{k}\right\}_{k=1}^{n}$ is an arbitrary non-overlapping domains such that

$$
a_{k} \in B_{k}, \quad B_{k} \subset \overline{\mathbb{C}}, \quad k=\overline{1, n} .
$$

Let

$$
g_{B}(B, a)=h_{B, a}(z)+\log \frac{1}{|z-a|}
$$

is the generalized Green's function of the domains $B$ with respect to a point $a \in B$. If $a=\infty$, then

$$
g_{B}(B, \infty)=h_{B, \infty}(z)+\log \frac{1}{|z|} .
$$

By the value

$$
r(B, a):=\exp \left(h_{B, a}(z)\right)
$$

wee define the inner radius of the domain $B \subset \overline{\mathbb{C}}$ with respect to a point $a \in B$ (see [4], [5], [6], [13], [14], [15]).

We use the concept of a quadratic differential. Recall that a quadratic differential on a Riemann surface $S$ is a map

$$
\varphi: T S \rightarrow \mathbb{C}
$$

satisfying

$$
\varphi(\lambda v)=\lambda^{2} \varphi(v)
$$

for all $v \in T S$ and all $\lambda \in \mathbb{C}$. If $z \in U \rightarrow \mathbb{C}$, is a chart defined on some open set $U \subset S$ then $\varphi$ is equal on $U$ to

$$
\varphi_{U}(z) d z^{2}
$$

for some function $\varphi_{U}$ defined on $z(U)$.

Suppose that two charts $z: U \rightarrow \mathbb{C}$ and $w: V \rightarrow \mathbb{C}$ on $S$ overlap, and let

$$
h:=w \circ z^{-1}
$$

be the transition function. If $\varphi$ is represented both as $\varphi_{U}(z) d z^{2}$ and $\varphi_{V}(w) d w^{2}$ on $U \cap V$, then we have

$$
\varphi_{V}(h(z))\left(h^{\prime}(z)\right)^{2}=\varphi_{U}(z) .
$$

One way to say this is that quadratic differentials transform under pull-backs by the square of the derivative. As the main results associated with it can be found in [16]. 


\section{Results}

Lemma. The function

$$
P(\tau)=\ln \sin \frac{\pi \tau}{2}
$$

is convex for $\tau \in(0,2)$.

Proof of Lemma. Find the second-order derivative

$$
P^{\prime \prime}(\tau)=\frac{\pi}{2} \cdot\left(\operatorname{ctg} \frac{\pi \tau}{2}\right)^{\prime}=-\left(\frac{\pi}{2}\right)^{2} \cdot \frac{1}{\sin ^{2} \frac{\pi \tau}{2}}
$$

Consequently,

$$
P^{\prime \prime}(\tau)<0 \text {, for } 0<\tau<2 \text {. }
$$

This proves the lemma.

Theorem. Let $n \in \mathbb{N}, n \geq 2$. Then for all system of the points $A_{n}=\left\{a_{k}\right\}_{k=1}^{n}$, which satisfy the condition (1), and an arbitrary set of the non-overlapping domains $\left\{B_{k}\right\}_{k=1}^{n}$ that satisfies the condition (2), the inequality holds

$$
\prod_{k=1}^{n}\left(\left|a_{k+1}-a_{k}\right|^{\alpha} \cdot r\left(B_{k}, a_{k}\right)\right) \leq\left(\frac{2^{\alpha+2}}{n} \cdot \sin ^{\alpha} \frac{\pi}{n}\right)^{n} .
$$

The equality is obtained in this inequality when points $a_{k}$ and domains $B_{k}$ are, respectively, the poles and the circular domains of the quadratic differential

$$
Q(w) d w^{2}=-\frac{w^{n-2}}{\left(w^{n}-1\right)^{2}} d w^{2} .
$$

As a consequence, at $\alpha=0$, we obtain the well known result of the V.N. Dubinina.

Corollary. [4], [5], [6]. Let $n \in \mathbb{N}, n \geq 2$. Then for all system of the points $A_{n}=\left\{a_{k}\right\}_{k=1}^{n}$, which satisfy the condition (1), and an arbitrary set of the non-overlapping domains $\left\{B_{k}\right\}_{k=1}^{n}$ that satisfies the condition (2), the inequality holds

$$
\prod_{k=1}^{n} r\left(B_{k}, a_{k}\right) \leq\left(\frac{4}{n}\right)^{n}
$$

The equality is obtained in this inequality, when the points $a_{k}$ and domains $B_{k}$ are, respectively, the poles and the circular domains of the quadratic differential (3).

Proof of Theorem. The proof of the theorem leans on a method of the piece-dividing transformation developed by Dubinin (see [4], [5], [6]).

The function

$$
\zeta_{k}(w)=-i\left(e^{-i \arg a_{k}} w\right)^{\frac{1}{\sigma_{k}}}, \quad k=1,2, \ldots, n
$$

realizes univalent and conformal transformations of the domain $M_{k}$ to the right half-plane $\operatorname{Re} \zeta>0$, for all $k=\overline{1, n}$.

From a formula (4) we receive the following asymptotic expressions

$$
\left|\zeta_{k}(w)-\zeta_{k}\left(a_{m}\right)\right| \sim \frac{1}{\sigma_{k}}\left|w-a_{m}\right|, w \rightarrow a_{m}, k=1,2, \ldots, n, m=k, k+1 .
$$

It's obvious that

$$
\zeta_{k}\left(a_{k}\right)=-i, \zeta_{k}\left(a_{k+1}\right)=i, k=1,2, \ldots, n \text {. }
$$


For the family of the functions $\left\{\zeta_{k}(w)\right\}_{k=1}^{n}$, setted by the equality (4), it is possible the piecedividing transformation (see [4], [5], [6]) of the domains $\left\{B_{k}: k=\overline{1, n}\right\}$ in relation to the system of the corners $\left\{M_{k}\right\}_{k=1}^{n}$. For any domain $\Delta \in \mathbb{C}$ we define $(\Delta)^{*}:=\{w \in \overline{\mathbb{C}}: \bar{w} \in \Delta\}$. Let $G_{k}^{(1)}$ means the connected component $\zeta_{k}\left(B_{k} \cap \bar{M}_{k}\right) \cup\left(\zeta_{k}\left(B_{k} \cap \bar{M}_{k}\right)\right)^{*}$, containing a point $(-i), G_{k-1}^{(2)}$ means the connected component $\zeta_{k-1}\left(B_{k} \bigcap \bar{M}_{k-1}\right) \bigcup\left(\zeta_{k-1}\left(B_{k} \bigcap \bar{M}_{k-1}\right)\right)^{*}$, containing a point $i, k=\overline{1, n}$, $\bar{M}_{0}:=\bar{M}_{n}, \zeta_{0}:=\zeta_{n}, G_{0}^{(2)}:=G_{n}^{(2)}$. It is clear, that, in general, $G_{k}^{(s)}$ domains are the multiconnected domains, $k=\overline{1, n}, s=1,2$. A pair of the domains $G_{k-1}^{(2)}$ and $G_{k}^{(1)}$ is grows out of the piece-dividing transformation domains $B_{k}$ concerning families $\left\{M_{k-1}, M_{k}\right\},\left\{\zeta_{k-1}, \zeta_{k}\right\}$ in point $a_{k}, k=\overline{1, n}$.

From the Theorem 1.9 [13] (see also [5], [6]) and the formula (5), we have the inequalities

$$
r\left(B_{k}, a_{k}\right) \leq\left[\sigma_{k} \cdot r\left(G_{k}^{(1)},-i\right) \cdot \sigma_{k-1} \cdot r\left(G_{k-1}^{(2)}, i\right)\right]^{\frac{1}{2}}, k=1,2, \ldots, n .
$$

From the condition that the points $a_{k}, k=1,2, . ., n$ are belong to the unit circle, we get that

$$
\left|a_{k+1}-a_{k}\right|=2 \sin \frac{\pi \sigma_{k}}{2}, k=1,2, \ldots, n .
$$

Using formulas (7), (8) we have:

$$
\begin{gathered}
\prod_{k=1}^{n}\left(\left|a_{k+1}-a_{k}\right|^{\alpha} \cdot r\left(B_{k}, a_{k}\right)\right) \leq 2^{n \alpha} \times \\
\times \prod_{k=1}^{n} \sin ^{\alpha} \frac{\pi \sigma_{k}}{2} \cdot \prod_{k=1}^{n}\left(\sigma_{k-1} \cdot \sigma_{k} \cdot r\left(G_{k}^{(1)},-i\right) \cdot r\left(G_{k}^{(2)}, i\right)\right)^{\frac{1}{2}} .
\end{gathered}
$$

Using the Lavrent'ev inequalities [1], we get:

$$
r\left(G_{k}^{(1)},-i\right) \cdot r\left(G_{k}^{(2)}, i\right) \leq 4, k=1,2, \ldots, n .
$$

Taking into account the last inequality, the expression (9) can be written as follows:

$$
\prod_{k=1}^{n}\left(\left|a_{k+1}-a_{k}\right| \cdot r\left(B_{k}, a_{k}\right)\right) \leq 2^{n(\alpha+1)} \cdot \prod_{k=1}^{n} \sigma_{k} \sin ^{\alpha} \frac{\pi \sigma_{k}}{2} .
$$

Also,

$$
\prod_{k=1}^{n} \sigma_{k} \leq\left(\frac{2}{n}\right)^{n}
$$

The equality can be obtained in this inequality, if and only if

$$
\sigma_{1}=\sigma_{2}=\ldots=\sigma_{n}=\frac{2}{n} .
$$

Then, we have:

$$
\prod_{k=1}^{n}\left(\left|a_{k+1}-a_{k}\right| \cdot r\left(B_{k}, a_{k}\right)\right) \leq\left(\frac{2^{\alpha+2}}{n}\right)^{n} \cdot \prod_{k=1}^{n} \sin ^{\alpha} \frac{\pi \sigma_{k}}{2} .
$$

The equality can be reached in this inequality when the points $a_{k}$ and domains $B_{k}$ are, respectively, the poles and the circular domains of the quadratic differential 


$$
Q(\zeta) d \zeta^{2}=\frac{d \zeta^{2}}{\left(\zeta^{2}+1\right)^{2}}
$$

From the Lemma we have the function $\alpha \ln \sin \frac{\pi \sigma_{k}}{2}$ is convex for $\sigma_{k} \in(0 ; 2), \alpha \geq 0$. Hence, when $\sigma_{k} \in(0 ; 2)$, then

$$
\frac{\alpha}{n} \cdot \sum_{k=1}^{n} \ln \sin \frac{\pi \sigma_{k}}{2} \leq \alpha \ln \sin \left(\frac{\pi}{2} \cdot \frac{1}{n} \sum_{k=1}^{n} \sigma_{k}\right) .
$$

Given that

$$
\sum_{k=1}^{n} \sigma_{k}=2
$$

we obtain

$$
\prod_{k=1}^{n} \sin ^{\alpha} \frac{\pi \sigma_{k}}{2} \leq \sin ^{n \alpha} \frac{\pi}{n}
$$

The equality obtain in this inequality, if and only if

$$
\sigma_{1}=\sigma_{2}=\ldots=\sigma_{n}=\frac{2}{n}
$$

Then from (10) using formulas (12) it is received the following ratio

$$
\prod_{k=1}^{n}\left(\left|a_{k+1}-a_{k}\right| \cdot r\left(B_{k}, a_{k}\right)\right) \leq\left(\frac{2^{\alpha+2}}{n}\right)^{n} \cdot \sin ^{n \alpha} \frac{\pi}{n} .
$$

The equality holds in this inequality, when the points $a_{k}$ and domains $B_{k}$ are, respectively, the poles and the circular domains of the quadratic differential (3). It is derived from the square of the quadratic differential (11) conversion using

$$
\zeta=-i w^{\frac{n}{2}}
$$

The theorem is proved.

\section{Acknowledgement.}

The author is grateful to Prof. A. Bakhtin for suggesting of the problems and the useful discussions.

\section{References}

[1] M.A. Lavrent'ev, On the theory of conformal mappings, Tr. Fiz.-Mat. Inst. Akad. Nauk SSSR, 5 (1934) 159-245. (in Russian)

[2] G.M. Goluzin, Geometric theory of functions of a complex variable, Nauka, Moscow, USSR, 1966. (in Russian)

[3] G.P. Bakhtina, Variational methods and quadratic differentials in problems for disjoint domains, PhD thesis, Kiev, Ukrainian SSR, 1975. (in Russian)

[4] A.K. Bakhtin, G.P. Bakhtina, Yu.B. Zelinskii, Topological-algebraic structures and geometric methods in complex analysis, Inst. Math. NAS Ukraine, Kiev, Ukraine, 2008. (in Russian)

[5] V.N. Dubinin Separating transformation of domains and problems of extremal division, Zap. Nauchn. Sem. Leningrad. Otdel. Mat. Inst. Ros. Akad. Nauk. 168 (1988) 48-66. (in Russian) 
[6] V.N. Dubinin, Method of symmetrization in the geometric theory of functions of a complex variable, Usp. Mat. Nauk. 49(1) (1994) 3-76.

[7] A.K. Bakhtin, Inequalities for the inner radii of nonoverlapping domains and open sets, Ukr. Math. J. 61(5) (2009) 716-733.

[8] A.K. Bakhtin, A.L. Targonskii, Extremal problems and quadratic differential, Nonlin. Oscillations. 8(3) (2005) 296-301.

[9] A.L. Targonskii, Extremal problems of partially nonoverlapping domains on a Riemann sphere, Dop. NAN Ukr. 9 (2008) 31-36. (in Russian)

[10] A. Targonskii, Extremal problems on the generalized (n; d)-equiangular system of points, An. St. Univ. Ovidius Constanta. 22(2) (2014) 239-251.

[11] A.L. Targonskii, Extremal problems for partially non-overlapping domains on equiangular systems of points, Bull. Soc. Sci. Lett. Lodz. 63(1) (2013) 57-63.

[12] A. Targonskii, I. Targonskaya, On the One Extremal Problem on the Riemann Sphere, International Journal of Advanced Research in Mathematics. 4 (2016) 1-7.

[13] V.N. Dubinin, Asymptotic representation of the modulus of a degenerating condenser and some its applications, Zap. Nauchn. Sem. Peterburg. Otdel. Mat. Inst. 237 (1997) 56-73. (in Russian)

[14] V.N. Dubinin, Capacities of condensers and symmetrization in geometric function theory of complex variables, Dal-nayka, Vladivostok, Russia, 2009. (in Russian)

[15] W.K. Hayman, Multivalent functions, Cambridge University, Cambridge, 1958.

[16] J.A. Jenkins, Univalent functions and conformal mapping, Springer, Berlin, 1958. 